\title{
The role of gut microflora in mucosal immunity
}

The microbiome is of recent interest in mucosal immunity. Dr Gary Huffnagle and colleagues at the University of Michigan have identified a variety of intestinal microflora members which can metabolically
communicate with host cells and regulate mucosal immunity. Accumulating evidence suggests that various metabolites produced by hosts and microbiome members and their crosstalk are more important than previously thought. Understanding and exploring these interactions may be .

ntercellular communication within the body is vital to ensure homeostasis, the body healthy and functioning. It is often achieved through ligand-receptor binding and signalling, either locally or systemically to distant sites. These interactions are true for both host and bacterial cells. Ligands can be proteins (peptides, growth factors, and cytokines) or non-proteins generated from metabolic pathways (modified lipids, nucleic acid derivatives, and small molecules). Interkingdom communication between host and cells of the microbiome occurs at a molecular level either through host cell metabolites release and subsequent sensing from microbial cells, or via

to host receptors to generate an and its interactions with host cells have been shown to play a significant role in mediating immunity and mucosal barriers, especially in the gastrointest and urogenital tract and upper and lower airways.

INTESTINAL EPITHELIUM STRUCTURE AND MUCOSAL IMMUNITY The intestinal epithelium within the gut is composed of epithelial cells adjoined by intercellular tight junctions (TSs), comprised of various proteins (occludins, claudins, mucins, etc) and junctiona adhesion molecules. Together, the epithelial cells and TJs create a dynamic

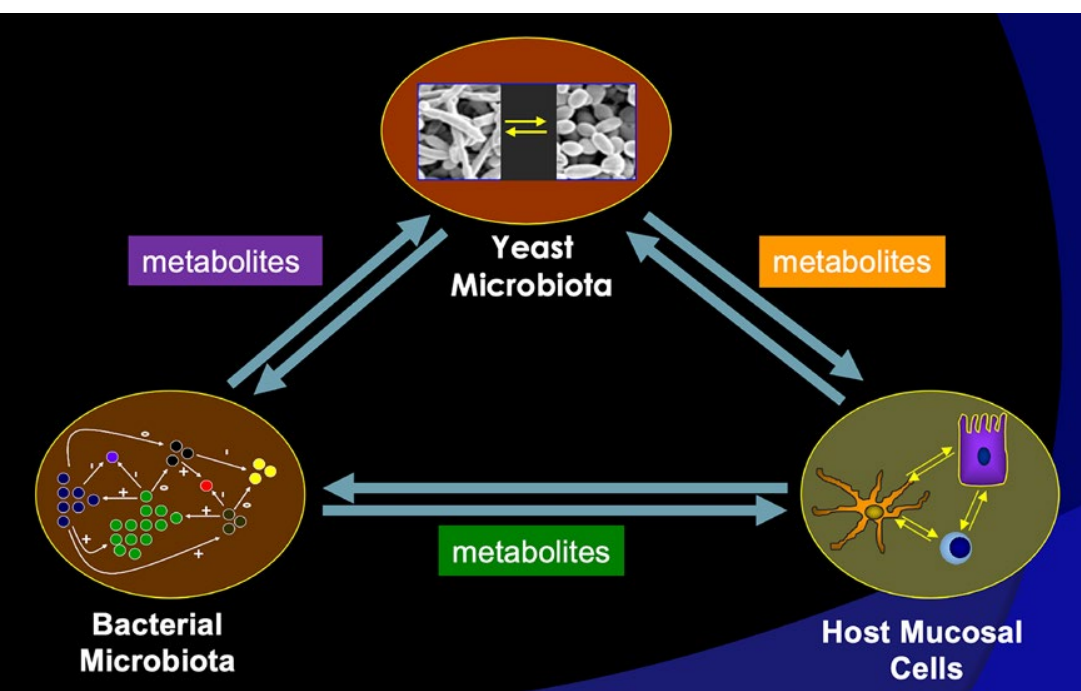

sective reabsorption of nutrients and Imits access to antigens and pathogens from deep within mucosal tissue. The microbiota resides on the apical surface of epithelial cells (which faces the inner surface of the gutt; they can also be found in the gut lumen which is the opening inside the bowels. The microbiome are critical regulators of gut integrity and can positively or negatively affect barier function by changing the function and/or expression of epithelial TJs.

Members of the microbiome interact with imm unoregulatory cells through the release of microbe-associated molecular pattern molecules which are recognised by immune cells through pattern

recognition receptors (PPRs). Antigenpresenting cells (APCs), including dendritic cells (DCs), macrophages, and $B$ cells, are modulated by numerous bacterial metabolites and trigger activation of the inflammasome - a collection of proteins that coordinate induction of inflammatory signals Signals also lead to the gen death. CD4 T regulary cells (Tregs) with and surrounding the intestinal mucosa. Tregs are important in regulating inflammation through the expression of the transcription factor (TF) called Foxp 3 which helps downregulate other T-help (Th) subsets involved in inflammation. Tregs also downregulate inflammation by secretion of transforming-growth factor $\beta$ and interleukin (LL)-10. Without Tregs, mucosal inflammation is enhanced by Th1, Th2, and Th17 cells that drive host inflammation.

Although mucosal immunity is thought to be largely mediated through host cell interactions, Dr Gary Huffnagle and colleagues at the University of Michigan
have identified microbial cells to be a bigger contributor in regulating mucosal immunity than first thought. They have ara of potentil immunomodulory molecules derived from the host or microbiome that facilitate this interaction and modulate mucosal immunity. These include short-chain fatty acids, prostaglandins, bile acids, uric acid, histamine, catecholamines, and ary hydrocarbon receptor ligands.

SHORT-CHAIN FATTY ACIDS Short-chain fatty acids (SCFAs), including butyrate and propionate, are metabolites produced by anaerobic bacteria within the microbiome of the large intestine. Propionate is also found in the intestin mucosa. These metabolites bind to epithellal cells or APCs expressing host

In host cells, butyrate and propionate promote intestinal epithelium survival and ncrease barrier integrity. Butyrate can act a major metabolic substrate for colon sunival by promoting cell differentiation and proliferation. Butyrate can also induce expression of mucin messenger RNA, which are the protein-coding instructions for assembly of mucin which compromise TJs, which therefore enhances barrier function. Butyrate and propionate have a wide variety of different effects on immune cells too. While butyrate can modulate differentiation, maturation, and function of $A P C$ s, propionate can alter $D C$ precursors rendering them less reactive with an impaired
Th-2 responses.

Researchers found that SCFA-producing clostridial species, which ape a bacterial species of the microbiome, can promote Treg accumulation in the intestine and surrounding tissue. Treatment of naiive $T$ cells with butyrate alone promote T-reg induction by blocking deacetylation of histone $\mathrm{H} 3$ promoter and conserved egions of the foxp3 locus, which is where the Foxp3 gene is. Propionate could also induce Treg development in the periphery. Interestingly, the SCFA receptor GPRTO9A is also a receptor for niacin (also produced by gut microbio PRTOA and niacin signalling was Phown to promocte antiming lam was differentiation of Tregs.

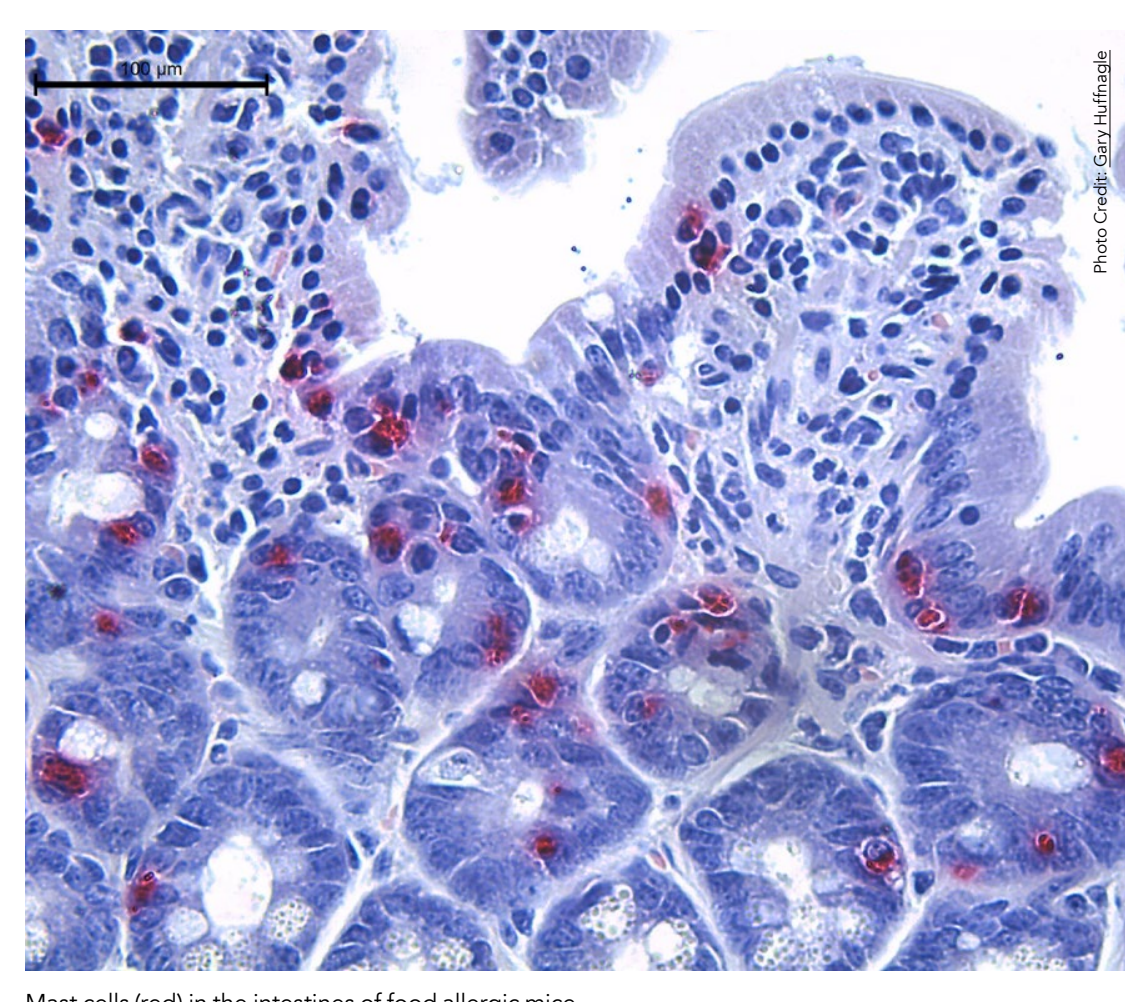

Dr Gary Huffnagle and colleagues have identified a wide array of potential immunomodulatory molecules derived from the microbiome.

PROSTAGLANDINS

Prostaglandins are lipids within host

tissue that are enzymatically generated

by synthases and other fatty acids. The

critical in regulating innate and adaptive immunity and epithelial function.

can also produce prostaglondins and

similar bioactive lipids (oxylipins)

which regulate host cell immune

cell activity similarly.

Researchers found that in a mouse model of allergy, pathogenic yeast Candida albicans produced high levels of PGE2 and oxylipins which promoted Th2-mediated inflammation and consequently decreased barrier function and increased permeability of intestinal epithelial cells. C. albican can also secrete a cytolytic peptide toxin called candidalysin which causes inflammation, formation of membran pores, and subsequent destruction also demonstated thasers prostaglandin production during funga infection could be important in aiding fungal colonisation and promoting understood about microb not much is f prostag andins and prostaglacionmediated regulation of mucosal ity it is an emerging field with much interest.

\section{BILE ACIDS}

cids are generated in the liver by cholesterol breakdown and then further metabolised by intestinal bacteria to produce secondary bile acids. Both primary and secondary bile acids can bind to various cell surface or nuclear receptors (FXR and GPBARI/TGRS) with different affinities Mice rate further signal cascades. Thu disease models infering thmation in role in controlling infammerito poten

Lithocholic acid, a secondary bile acid, was shown to inhibit IL-1 $\beta$ and 


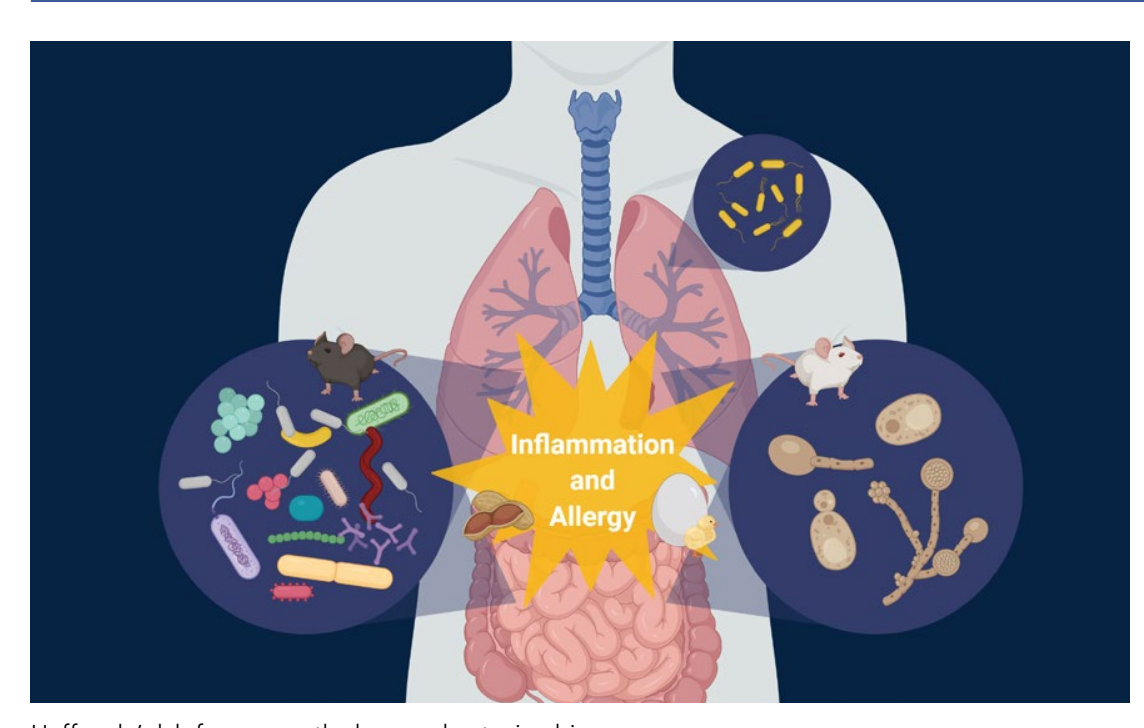

Uuffragle's lab focuses on the lung and gut microbiome

NLRP3 inflammasome assembly by macrophages via TGR5 signalling degrabiquition affecting the macrophages destruction capabilities. Another microbial-derived secondary bile acid called 3-OxolCA can repress Th17 cell activity by inhibiting Th17 differentiation via secretion of IL-22, IL-17, and others. This is important as Th17 cells are critical for barrier maintenance of gut epithelial cells.

URIC ACID Uric acid is produced by host cells from HISTAMIN Histamine is a vasoactive biogenic binds to hista rom hister which binds to histamine receptors and plays tissue fluid movement, seep, and gastic acid secretion. Histamine can induce oedema within the submucosal tissue below the intestinal epithelium causing fluid movement out of the tissue without compromising the integrity of $\mathrm{TJs}$. Despite its well-known role in host cel biology, little is known about bacteriaderived histamine. However, there is increasing evidence that bacterialderived histamine within the microbiota

There is increasing evidence that bacterialderived histamine within the microbiota plays a role in mucosal immunity as there are many histamine-secreting microbes within the human gut microbiome.

various host binding proteins. Uric acid can also activate various proteins and pathways associated with the NLRP3 inflammasome and cell death. Bacterial cells of the microbiota can also produce model, researer pathways. In a colltis aid intestinal colonisation of the yeast Saccharomyces cerevisiae which further enhances host purine metabolism and uric acid production in intestina epithelial cells. Uric acid treatment was function by increasing gut permer

are many histamine-secreting microbes within the human gut microbiome.

Histamine-secreting Lactobacillus species were described over 50 years ago, and accumulative evidence sugges bacterial-derived histamine plays a protective role in downregulating Microbiome-derived histamine is thoug to act through NLRPG inflammason signalling, I 18 secretion and antimicrobial peptide production and be
S inflammatory cytokine production.

CATECHOLAMINES

Catecholamines, another class

enzymatically by phenylalanine

or tyrosine to create epinephrine

norepinephrine, and dopamine. These

molecules act on various dopamine

and adrenergic receptors. Molecules

can act as a hormone systemically

.

Macrophages have also been seen to

augment innate inflammation by their beal release of catecholamines. Similarly bacterial-derived catecholamines can mediating bacterial pathogenic pathways

\section{ARYL HYDROCARBON}

Aryl hydrocarbon receptor (AHR) is a

IF and cytoplasmic receptor, serving

as a receptor for many small molecules

to induce $A H R$ activation. AHR ligands

include host-derived tryptophan

metabolites and other AHR ligands

provided by the members of the

intestinal microbiome. AHR ligands

can regulate aspects of mucosal and systemic immunity and modulate host-

. . derived fromyde, an AHR ligand

dietary trytophan con malsm

gut Peyer's patches cells to previ-22 in fungal resistances by mediated inflammation.

\section{CONCLUSIONS}

Host cells and the microbiome

act via ligand-recepto interactions and can regulate mucosa mmunity and inflammation, which is important for further understanding mucosal immunity. However, attributing the role of either host microbial-derived immunomodulatory metabolites is difficult when other contributing factors affect mucosal immunity, including diet, genetics, and environmental factors. Despite this, researchers state that metabolic crosstalk between kingdoms is crucial in providing a deeper understanding of mucosal immunity and allergens, food, xenobiotic, and disease.

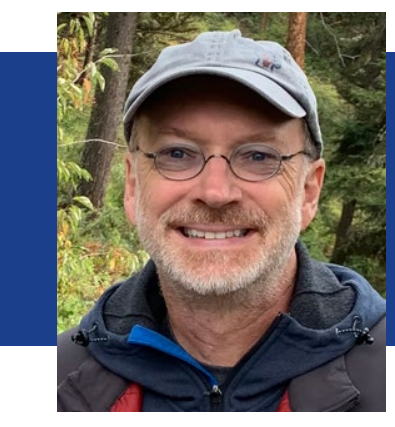

\section{Behind the Research} Dr Gary B Huffnagle

E: ghuff@umich.edu T: +17347646994 (office) T: +17347647427 (department)

Research Objectives

Dr Huffnagle and his lab study the lung and gut microbiome and its role in the manifestation of respiratory and food allergies.

\section{Detail}

Address

3232 Biological Sciences Building

1105 N University Ave.

Ann Arbor, MI 48109-1085, USA

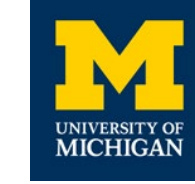

Gary B Huffnagle, PhD is a Professor of Internal Medicine, Developmental Biology at the University of Michigan. also holds the Nina and Jerry D Luptak Professorship. Dr Huffnagle received his $\mathrm{PhD}$ in immunology from the

University of Texas Southwestern Medical School and was elected to the American Academy of Microbiology of the American Society for Microbiology in 2013. Dr Huffnagle has been a frequent reviewer for the National Instiutes of Health, including standing membership for multiple study ternational lectures and talks.

Dr Huffnagle's research interests for the past three decades include the interaction between (1) opportunistic fungal pathogens, (2) the microbiome and (3) the immune system during inflammatory and allergic diseases of the lungs, gastrintestinal tract and multi-organ systems (anaphylaxis). microbing focus of his laboratory is the role of the gut food allergies.

Funding

- National Institute of Allergy and Infectious Diseases - National Heart, Lung and Blood Institute r Food Allergy Center

Collaborators

Robert Dickson, Fernando Martinez, Yvonne Huang, Jeff Curtis, John LiPuma, Meilan Han, Nick Lukacs, James Bake Ptaschingki, Nicole Falkowski, Kelsey Stark, Malak Baza, Ktaschinski, Nicole Falkowski, Kelsey Stark, Malak Bazzi, Baker, Alex Ethridge, Charles Schuler, IV

\section{References}

- Ethridge, AD, Bazzi, MH, Lukacs, NW, et al, (2021) Interkingdom Communication and Regulation of Mucosal Immunity by the Microbiome. The Journal of Infectious Diseases, 223(3) 236-240. doi.org/10.1093/infdis/iiaa748

- Dickson, RP, Erb-Downward, JR, Matinez, FJ, et al, (2016) The Microbiome and the Respiratory Tract. Annual Review of Physiology, 78(8), 481-504. doi.org/10.1146/annurevphysiol-021115-105238

- Scales, BS, Dickson, RP, Huffnagle, GB, (2016) A tale of two gut and lungs. Journal of Leukocyte Biology, 100, 943-950 do org/10.1189//1b.3mr0316-106r - Noverr, MC, Falkowski, NR, Mc Development of Allergic Aimway Disease in Mice foos) Antibiotic Therapy and Fungal Microbiota Increase: Role of Host Genetics, Antigen, and Interleukin-13. Infection and Immunity, 73(1), 30-38. doi.org/10.1128/Al.73.1.30-38.2005 - Noverr, MC, Noggle, RM, Toews, GB, et al, (2004) Role of antibiotics and fungal microbiota in driving pulmonary allergic responses. Infection and Immunity, 72, 4996-5003. doi,

org/10.1128/A.72.9.4996-5003.2004 - Noverr, MC, Phare, SM, Toews GB, et al, (2001) Pathogenic yeasts Cryptococcus neoformans and Candida albicans produce 2957-2963. do 257-2963. doi.org/10.1128/iai.69.5.2957-2963.2001

\section{Personal Response}

\section{How could this knowledge be applied clinically?}

II Small molecules (such as those derived from the

microbiome) can be easily synthesised in the laboratory and is possible. Regulatory agencies in Europe and North America are far more likely to approve these for clinical trials than whole, live bacteria. If we can identify microbiome-derived target molecules that dampen inflammation and provide strong pre-clinical data on their effectiveness, that will open significant therapeutic potentials for the treatment of food allergies, asthma, irritable bowel syndrome, inflammatory

Is this research true in other mucosal surfaces and their

residing microflora?

evolved to be maximally effective whal immune system has the normal microbiome. 